\title{
Direct Curvature Scale Space in Corner Detection
}

\author{
Baojiang Zhong ${ }^{1}$ and Wenhe Liao $^{2}$ \\ ${ }^{1}$ Department of Mathematics, Nanjing university of Aeronautics \\ \& Astronautics, Nanjing, 210016, China \\ zhbj@nuaa.edu.cn \\ ${ }^{2}$ College of Mechanical and Electrical Engineering, Nanjing university of Aeronautics \\ \& Astronautics, Nanjing, 210016, China \\ njwho@nuaa.edu.cn
}

\begin{abstract}
Curvature Scale Space (CSS) representation of planar curves is considered to be a modern tool in image processing and shape analysis. Direct Curvature Scale Space (DCSS) is defined as CSS that results from convolving the curvature of a curve with a Gaussian kernel directly. Recently a theory of DCSS in corner detection has been established. In the present paper the DCSS theory is considered to transform the DCSS image of a given curve into a tree organization, and then corners on the curve are detected and located in a multiscale sense. Experiments are conducted to show that the DCSS corner detector can work equally well as the CSS corner detector does on curves with multiple-size features, however, at much less computational cost.
\end{abstract}

\section{Introduction}

The scale space concept was introduced by Iijima [3] more than 40 years ago and became popular later on by the works of Witkin [13] and Koenderink 4]. Scale space analysis of a signal $f(x)$ is generally made by convolving it with a Gaussian kernel, treating the quadratic variance $\sigma$ of Gaussian as a parameter of scale. Extrema in the first derivative, or, zero-crossings in the second derivative of the convolved signal are located at varying scales. The image on the $(x, \sigma)$ plane showing the extrema or zero-crossings is called a scale space image.

Asada and Brady [1] extended the scale space concept to represent significant changes in curvature along a planar curve. The curve is expressed as a function $\varphi(s)$ of the orientation of the tangent $\varphi$ against arc length $s$. Then $\varphi(s)$ is convolved with a Gaussian. Local positive maxima and negative minima in the first and second derivatives of the convolved function are located, resulting two scale space images on the $(s, \sigma)$ plane. A set of curvature changes were selected as primitives. The scale space behavior of Corner and Smooth join was studied, and the behavior of other curvature primitives was illustrated.

Mokhtarian and Mackworth [56] developed Curvature Scale Space (CSS) by finding curvature zero-crossings of a curve at varying levels of detail. They 
treated the curve as a 2-D signal. Considering a path length variable $u$, the curve is expressed in terms of two functions: $\{x(u), y(u)\}$. Then the two functions are convolved respectively. By encoding the curve with the curvature zero-crossings, a multiscale shape representation was formulated. The CSS representation has been selected as a shape contour descriptor for MPEG-7 [7].

Rattarangsi and Chin [10] employed CSS to detect corners on planar curves. A CSS image consisting of the maxima of absolute curvature is constructed. The scale space behavior of isolated single and double corner models was sketched, with which the CSS image is transformed into a tree organization and then corners are detected. Pei and Lin [9] also proposed a corner detector based on the scale space concept. Similar to Asada and Brady's representation, they treated planar curves as 1-D signals. Extreme curvature points are located by convolving the curvature of a curve directly. Pei and Lin studied a procedure of corner detection. However, no scale space analysis was provided.

During the last decade, scale space concept has attracted a wide interest in the field of shape representation, feature extraction, and object recognition, see, e.g. 28111214 1516]. For distinguishing purpose, CSS resulting from direct curvature convolution is referred to as Direct Curvature Scale Space (DCSS). Note that since differentiation and convolution are commutative, Asada and Brady's representation relates to DCSS directly. Recently, the CSS theory in corner detection is re-established, and a DCSS theory is established [18. The problem of how a planar curve shrinks in its scale space was also mathematically studied in [18.

Compared to CSS, DCSS is much cheaper in terms of computational cost. Based on the DCSS theory, we study a procedure of DCSS corner detection in a multiscale sense. In Section 2 the DCSS theory is reviewed. In Section 3 the procedure of DCSS corner detection is presented and experiments are conducted. Finally, in Section 4 the paper is concluded.

\section{A Theory of Direct Curvature Scale Space}

Corners of a planar curve correspond to points of high curvature. Let $\kappa(s)$ be the curvature function. The DCSS representation describes the curve at increasing levels of detail by convolving $\kappa(s)$ with a Gaussian $g(s, \sigma)$ directly. Denote by $\otimes$ the convolution operator. The convolved curvature function $\kappa(s, \sigma)$ is given by

$$
\kappa(s, \sigma)=\kappa(s) \otimes g(s, \sigma) .
$$

It can be shown explicitly as

$$
\kappa(s, \sigma)=\frac{1}{\sigma \sqrt{2 \pi}} \int_{-\infty}^{+\infty} \kappa(u) e^{-\frac{(s-u)^{2}}{2 \sigma^{2}}} d u .
$$

$\overline{{ }^{1} \text { Rattarangsi }}$ and Chin presented a theoretical study of the CSS representation in corner detection; however, due to a fundamental mistake in their work the CSS theory was not established correctly. 
To determine corners at a given scale $\sigma$, we solve for all the locations that have maxima absolute curvature, $|\kappa(s, \sigma)|$, which are the positive maxima and negative minima of the curvature. A DCSS image of corners is then constructed by $\max _{s, \sigma}|\kappa(s, \sigma)|$. To investigate the properties of the DCSS image, the single corner $\Gamma$ model and double corner END and STAIR models [110] are considered.

Figure 1 shows a $\Gamma$ corner model and its DCSS image. The model properties are summarized in Property 1.

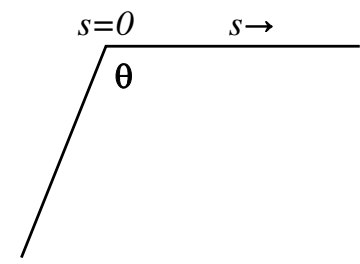

(a) $\Gamma$ model

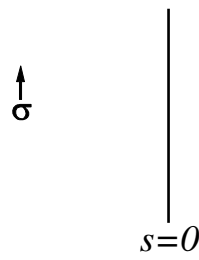

(b) DCSS image

Fig. 1. $\Gamma$ model produces a stationary and persistent scale space image

Property 1: A $\Gamma$ corner model has a single stationary and persistent line pattern in direct curvature scale space independent of the corner angle $\theta$ and the scale parameter $\sigma$.

Consider an END corner model, which consists of two corner $\theta_{l}$ and $\theta_{r}$ with the same concavity separated by a width of $2 w$, see Figure 2(a). Define

$$
\lambda=\frac{\pi-\theta_{r}}{\pi-\theta_{l}} ; s_{l}=\frac{\lambda-1}{\lambda+1} w .
$$

Let $s_{r}$ be implicitly given by

$$
\ln \left(\lambda \frac{w-s_{r}}{w+s_{r}}\right)+\frac{2 w s_{r}}{\left(w+s_{r}\right)\left(w-s_{r}\right)}=0, s_{r} \in(0, w),
$$

and define

$$
\sigma_{\lambda}^{2}=\left(w+s_{r}\right)\left(w-s_{r}\right)
$$

Properties of the END corner model are summarized in Property 2-4 and its DCSS image is sketched in Figure 2(b)

Property 2: For an END model with $0<\theta_{l}<\theta_{r}<\pi$, the DCSS line pattern of the strong corner $\theta_{l}$ is persistent and asymptotically stationary at $s=s_{l}$.

Property 3: For an END model with $0<\theta_{l}<\theta_{r}<\pi$, the DCSS line pattern of the weak corner $\theta_{r}$ terminates at $\left(s_{r}, \sigma_{\lambda}\right)$, and there it meets another kind of line pattern which consists of a set of minima of absolute curvature.

Property 4: An END model, with a corner separation $2 w$ and $0<\theta_{l}=\theta_{r}<\pi$, has a DCSS image symmetric with respect to $s=0$. When $0<\sigma<w$, the two absolute maxima move towards each other as $\sigma$ increases. When $\sigma \geq w$, the two absolute maxima merge, forming a single stationary and persistent line pattern. 


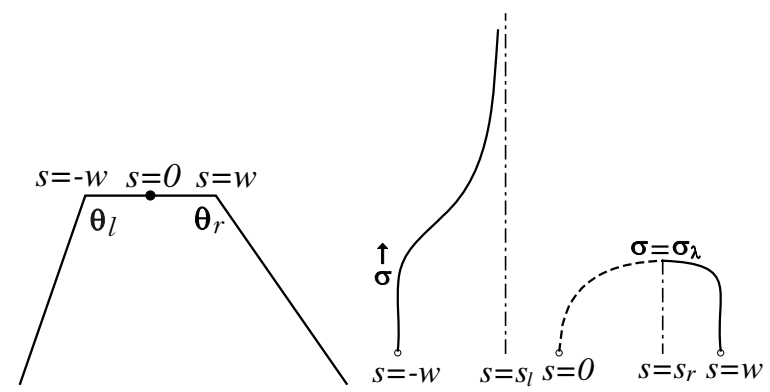

(a) END model

(b) DCSS image

Fig. 2. END model and its DCSS image

Consider a STAIR corner model, which consists of two corner $\theta_{l}$ and $\theta_{r}$ of opposite concavity separated by a width of $2 w$, see Figure 3(a). Its model properties are summarized in Property 5-7, and its DCSS image is sketched in Figure 3(b), Property 5: For a STAIR model with $0<\theta_{l}<2 \pi-\theta_{r}<\pi$, the DCSS line pattern of the strong corner $\theta_{l}$ is persistent and asymptotically stationary at $s=s_{l}$.

Property 6: For a STAIR model with $0<\theta_{l}<2 \pi-\theta_{r}<\pi$, the DCSS line pattern of the weak corner $\theta_{l}$ is persistent, and it is bounded by $\sigma=\mu \sqrt{s}$ as $\sigma$ increases, where $\mu$ is defined as $\mu=\left(-\frac{2 w}{\ln (-\lambda)}\right)^{1 / 2}$.

Property 7: A STAIR model with $0<\theta_{l}=2 \pi-\theta_{r}<\pi$ produces a persistent DCSS image, which is symmetric with respect to $s=0$. The two line patterns are bounded by $\sigma=-s$ and $\sigma=s$ respectively and they repel each other at a same rate as $\sigma$ increases.

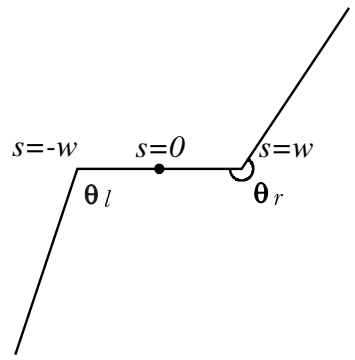

(a) STAIR model

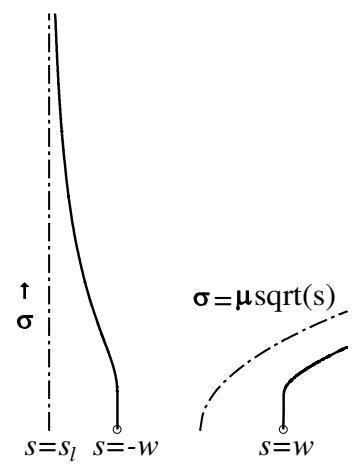

(b) DCSS image

Fig. 3. STAIR model and its DCSS image

To investigate the effect of corner separation of a double corner model on its DCSS image, we fix $\theta_{l}$ and $\theta_{r}$, and vary $w$. The following property is established. Property 8: The DCSS image of an END or STAIR model with corner separation $2 w_{i}$ is linearly related to the DCSS image of another END or STAIR model with corner separation $2 w_{j}$ by the ratio of the separations given by $\frac{w_{i}}{w_{j}}$. 


\section{DCSS Corner Detection}

To detect corners on a planar curve, a tree organization similar to that in [13]10]11] is constructed from its DCSS image. To filter out quantization noise,
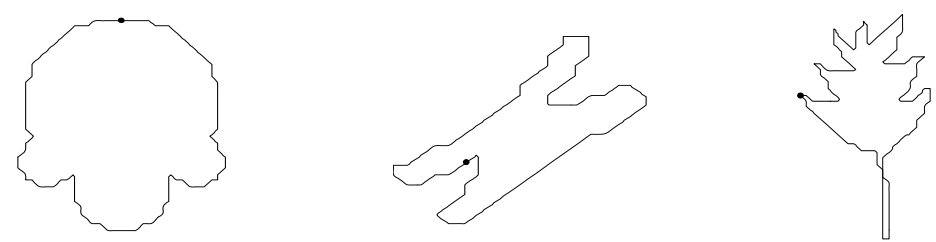

(a) Test curves: in contour-clockwise; start point is indicated by a bullet
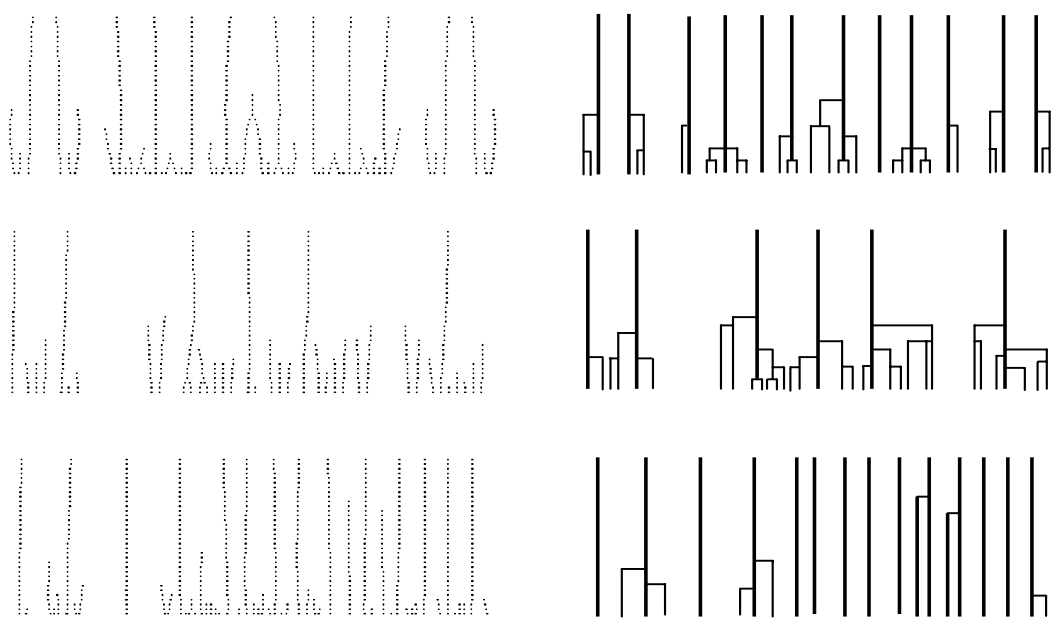

(b) DCSS images



(d) Corner detection results of DCSS
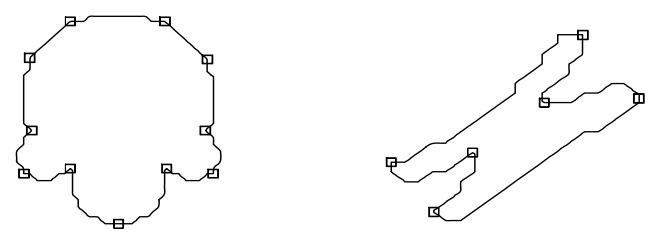

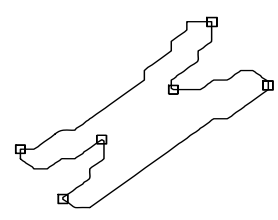

(c) Tree organizations

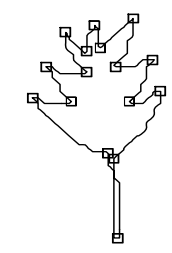

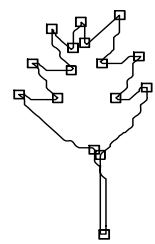

(e) Corner detection results of CSS

Fig. 4. DCSS corner detection and a comparison with CSS 
line patterns caused by extremely low absolute maxima (say, below 0.01) are removed from the image at first. After this cleaning process, for each line pattern a vertical line located at the finest scale location of the line pattern is drawn. If two line patterns merge to become a single one, two vertical lines are drawn respectively, and a single vertical line located at the merging scale location is also drawn. The three vertical lines are joined by a horizontal line. For a non-survival line pattern, the vertical line is joined by a horizontal line to the nearest more persistent vertical line.

The model properties specified in Section 2 are then considered to parse the tree organization. A tree consisting of a single root corresponds to a single corner located at the finest scale location of the root. For a tree with offspring, the length of its root is compared with the height of its offspring. If the root length exceeds the height of the offspring, the root is declared stable, corresponding to a corner located at the orthogonal projection of the root on $x$-axis. Otherwise the search for corners proceeds to the offspring. If an offspring is a leaf whose length is longer than that of its parent, it is stable, corresponding to a corner located at the finest scale location of the leaf. If an offspring has its own offspring, the search for corners is applied to its family. Each tree of the organization is evaluated in the same manner.

A set of objects that have been commonly used in many previous studies are chosen as test curves. They are the Semicircles, the Chromosome, and the Leaf, see Figure 4(a) (Since DCSS is invariant under rotation, the start point of each curve is selected randomly). Figure 4(b) shows their DCSS images, and Figure 4(c) shows the corresponding tree organizations. The roots and leaves of the trees which correspond to corner points are indicated by bold lines. Corner detection results of DCSS are shown in Figure 4(d).

For comparison, Figure 4(e) shows the results of CSS corner detection. It can be seen that the DCSS corner detector compares favorably with CSS. For the last two curves, the two detectors have the same performance. For the first curve, we believe the performance of the DCSS detector is more reasonable: the semicircles with different radii have been distinguished by different corner patterns.

On the other hand, the DCSS corner detector outperforms the CSS corner detector very clearly with respect to computational cost. Table 1 summarizes a comparison between the CPU processing time of the two detectors spent on the three curves. The programs were implemented on a Pentium IV-2.80G PC with $512 \mathrm{M}$ memory. It can be seen that the DCSS detector requires only about $1 / 3$ CPU time of the CSS detector. As a result, the efficiency of corner detection is improved significantly.

Table 1. Comparison of the CPU time of the CSS and DCSS detectors

\begin{tabular}{cccc}
\hline \multicolumn{4}{c}{ semicircle curve chromosome curve leaf curve } \\
\hline CSS & $166 \mathrm{~ms}$ & $190 \mathrm{~ms}$ & $186 \mathrm{~ms}$ \\
DCSS & $57 \mathrm{~ms}$ & $63 \mathrm{~ms}$ & $62 \mathrm{~ms}$ \\
\hline
\end{tabular}




\section{Concluding Remarks}

In this paper we have studied a procedure of applying DCSS to detect and locate corners on planar curves. Numerical results show that the DCSS corner detector can operate successfully on curves with multiple-size features, however, at much less computational cost compared to the CSS corner detector.

To compute a scale space image, DCSS is much cheaper than CSS. This can be appreciated by a glance at the computational complexity of the two representations.

CSS: Compute $\{x(s, \sigma), y(s, \sigma)\}$ and the curvature of the convolved curve at each scale;

DCSS: Compute the curvature at scale $\sigma=0$, and compute $\kappa(s, \sigma)$ at each scale.

Since DCSS computes only one scale space instead of two, and computes the curvature of the curve (an expensive operation) only once, its computational cost is significantly less than that of CSS.

For noisy curves, a small amount of Gaussian smoothing is suggested to be a preprocessing step of DCSS [9]. In particular, during the smoothing process absolute maxima in curvature can be located as a by-product, and then a small part of the CSS image can be constructed. This brings to us a hybrid scheme to apply CSS and DCSS [17, by which corners can be located at the finest scale. The hybrid CSS/DCSS corner detector, including a switchover criterion between CSS and DCSS, has be discussed in detail in [18].

\section{References}

1. Asada, H., Brady, M.: The curvature primal sketch. IEEE Trans. Pattern Anal. Mach. Intell. 8 (1986) 2-14

2. Garrido, A., Blanca, N., Vente, M.: Boundary simplification using a mutiscale dominant-point detection algorithm. Pattern Recognition 31 (1998) 791-804

3. Iijima, T.: Basic theory on normalization of pattern (in case of typical onedimensional pattern). Bulletin of the Electrotechnical Laboratory 26 (1962) 368388

4. Koenderink, J.J.: The structure of images. Biol. Cybern. 50 (1984) 363-370

5. Mokhtarian, F., Mackworth, A.: Scale-based description and recognition of planar curves and two-dimensional shapes. IEEE Trans. Pattern Anal. Mach. Intell. 8 (1986) 34-43

6. Mokhtarian, F., Mackworth, A.: A theory of multi-scale, curvature-based shape representation for planar curves. IEEE Trans. Pattern Anal. Mach. Intell. 14 (1992) 789-805

7. Mokhtarian, F., Bober, M.: Curvature Scale Space Representation: Theory, Applications, and MPEG-7 Standardization. Kluwer Academic Publishers, Dordrecht, March 2003.

8. Mokhtarian, F., Abbasi, S.: Robust automatic selection of optimal views in multiview free-form object recognition. Pattern Recognition 38 (2005) 1021-1031 
9. Pei, S., Lin, C.: The detection of dominant points on digital curves by scale-space filtering. Pattern Recognition 25 (1992) 1307-1314

10. Rattarangsi, A., Chin, R.: Scale-based detection of corners of planar curves. IEEE Trans. Pattern Anal. Mach. Intell. 14 (1992) 430-449

11. Ray, B., Ray, K.: Corner detection using iterative Gaussian smoothing with constant windows size. Pattern Recognition 281995 1765-1781

12. Ray, B., Pandyan, R.: ACORD-an adaptive corner detector for planar curves. Pattern Recognition 36 (2003) 703-708

13. Witkin, A.P.: Scale-space filtering. In: Proc. Eighth Int. Joint Conf. on Artificial Intelligence, Karlsruhe, Germany (1983) 1019-1021

14. Xin, K., Lim, K., Hong, G.: A scale-space filtering approach for visual feature extraction. Pattern Recognition 28 (1995) 1145-1158

15. Zabulis, X., Sporring, J., Orphanoudakis, S.: Perceptually relevant and piecewise linear matching of silhouettes. Pattern recognition 38 (2005) 75-93

16. Zhang, D., Lu, G.: Review of shape representation and description techniques. Pattern Recognition 37 (2004) 1-19

17. Zhong, B.J., Liao W.H.: A hybrid method for fast computing the curvature scale space image. International Conference on Geometric Modeling and Processing, IEEE Computer Society Press (2004) 124-130

18. Zhong, B.J.: Research on Algorithms for Planar Contour Processing. PhD Dissertation, Nanjing university of Aeronautics \& Astronautics (2006) 